\title{
BOUNDARY BEHAVIOR OF HARMONIC FUNCTIONS ON HERMITIAN HYPERBOLIC SPACE ${ }^{1}$
}

\section{BY ROBERT PUTZ}

Communicated by J. J. Kohn, May 11, 1970

Let $D=\left\{z=\left(z_{1}, \cdots, z_{n}\right) \in C^{n}: h(z)=\operatorname{Im} z_{1}-\sum_{2}^{n}\left|z_{k}\right|^{2}>0\right\}$, and $B=\partial D=\{z: h(z)=0\}$. Writing $z_{j}=x_{j}+i y_{j}$ we let $\beta$ be the measure on $B$ given by $d \beta=d x_{1} d x_{2} d y_{2} \cdots d x_{n} d y_{n} . D$ is a Siegel domain of Type II which is the image of the unit ball $D=\left\{z \in C^{n}: \sum_{1}^{n}\left|z_{k}\right|^{2}<1\right\}$ under the generalized Cayley transform:

$$
z_{1} \mapsto i \frac{1+z_{1}}{1-z_{1}}, \quad z_{k} \rightarrow \frac{i z_{k}}{1-z_{1}}, \quad k=2, \cdots, n .
$$

Let $N$ be the group of holomorphic automorphisms of $D$ consisting of the elements $(a, c) \in R \times C^{n-1}$ acting on $D$ in the following way:

$$
\begin{aligned}
& (a, c): z_{1} \rightarrow z_{1}+a+2 i \sum_{k=2}^{n} z_{k} \bar{c}_{k}+i \sum_{k=2}^{n}\left|c_{k}\right|^{2}, \\
& (a, c): z_{k} \rightarrow z_{k}+c_{k}, \quad k \geqq 2 .
\end{aligned}
$$

$N$ acts simply transitively on $B$. We will consider real-valued functions on $D$ which are harmonic with respect to the Laplace-Beltrami operator:

$L=h(z)\left\{4 y_{1} \frac{\partial^{2}}{\partial z_{1} \partial \bar{z}_{1}}+\sum_{2}^{n} \frac{\partial^{2}}{\partial z_{k} \partial \bar{z}_{k}}+2 i \sum_{2}^{n} \bar{z}_{k} \frac{\partial^{2}}{\partial z_{1} \partial \bar{z}_{k}}-2 i \sum_{2}^{n} z_{k} \frac{\partial^{2}}{\partial \bar{z}_{1} \partial z_{k}}\right\}$.

In [2] Korányi defined the following notion of admissible convergence in $D$ : let us call

$$
\begin{aligned}
\Gamma_{\alpha}(u)=\left\{z \in D: \operatorname{Max}\left[\left|\operatorname{Re} z_{1}-\operatorname{Re} u_{1}\right|, \sum_{2}^{n} \mid z_{k}\right.\right. & \left.-\left.u_{k}\right|^{2}\right] \\
& <\alpha h(z), h(z)<1\}
\end{aligned}
$$

AMS 1969 subject classifications. Primary 3111, 3210; Secondary 2270.

Key words and phrases. Hermitian hyperbolic space, Laplace-Beltrami operator, admissible convergence, harmonic functions, area integral.

1 This contains a summary of results in the author's $\mathrm{Ph}$. D. dissertation at Washington University written under the direction of Professor R. R. Coifman. I take pleasure in thanking Professor Coifman for his valuable assistance, and Professor Guido Weiss for his advice and encouragement. 
a truncated admissible domain of aperture $\alpha$ at $u \in B$. We say that $f$ on $D$ converges admissibly at $u$ to $l$ if $\lim _{z \rightarrow u ; z \in \Gamma_{\alpha}(u)} f(z)=l$, for some $\alpha>0$.

The principal result of this note is the Theorem below, which is the analogue of results of Marcinkiewicz and Zygmund [3], Spencer [4], Calderón [1], and Stein [5]. (This is often referred to as the Area theorem for harmonic functions.) Let

and

$$
\nabla f=\left(2 h^{1 / 2} \frac{\partial f}{\partial z_{1}}, 2 i \bar{z}_{2} \frac{\partial f}{\partial z_{1}}+\frac{\partial f}{\partial z_{2}}, \cdots, 2 i \bar{z}_{n} \frac{\partial f}{\partial z_{1}}+\frac{\partial f}{\partial z_{n}}\right)
$$

$$
|\nabla f|^{2}=4 h\left|\frac{\partial f}{\partial z_{1}}\right|^{2}+\sum_{2}^{n}\left|2 i \bar{z}_{k} \frac{\partial f}{\partial z_{1}}+\frac{\partial f}{\partial z_{k}}\right|^{2} .
$$

Let $E$ be a measurable set in $B$ and suppose that $f$ is a real-valued harmonic function in $D$.

TheOREm. (a) If $f$ is admissibly bounded for each point of $E$ then

$$
\int_{\Gamma_{\alpha}(u)} h(z)^{-n}|\nabla f|^{2} d \mu(z)<\infty
$$

for almost every $u$ in $E$ and $\alpha>0$, where $d \mu$ is Lebesgue measure.

(b) If, for each point $u$ of $E$, we can find an $\alpha$ such that the integral (1) is finite, then $f$ converges admissibly at almost every point of $E$.

The general outline of the proof follows Stein [5]. The differences arise from the fact that the Laplace-Beltrami operator is not uniformly elliptic. We first indicate how part (a) is proved. By a standard argument (see Calderón [1]) we may assume that $E$ is compact, and $f$ is uniformly bounded in $\Gamma_{\alpha}(u)$, for $\alpha$ fixed, and all $u \in E$.

LEMMA 1. If $f$ is bounded and harmonic in $\Gamma_{\alpha}(0)$, then $h(z)\left|\partial f / \partial z_{1}\right|$ and $h(z)^{1 / 2}\left|\partial f / \partial z_{k}\right|, k \geqq 2$, are bounded in $\Gamma_{\alpha^{\prime}}(0)$ for $\alpha^{\prime}<\alpha$.

This result can be proved by using the Poisson integral representation for functions defined on images of spheres under the Cayley transform.

Let $\omega_{\alpha}(E)=\cup_{u \in E} \Gamma_{\alpha}(u)$. We construct regions approximating $\omega_{\alpha}(E)$. Write $z \in D$ as $z=[x, \tilde{z}]_{t}$ where $x=x_{1}, \tilde{z}=\left(z_{2}, \cdots, z_{n}\right), t=h(z)$. Sirte $E$ is compact, $E_{t}=\left\{[x, \tilde{z}]_{t}:[x, \tilde{z}]_{0} \in E\right\}$ is compact. For $0<t<1$ let $\Gamma_{\alpha}(u)_{t^{2}}=\left\{\left[\begin{array}{ll}x, & \tilde{z}\end{array}\right]_{r+t^{2}}:\left[\begin{array}{ll}x, & \tilde{z}\end{array}\right]_{r} \in \Gamma_{\alpha}(u)\right.$ and $\left.r+t^{2}<1\right\}$. Then $\left\{\Gamma_{\alpha}(u)_{t}{ }^{2} \cap E_{t}\right\}_{u \in E}$ forms an open cover of $E_{t}$. Choose a finite subcover for $t=t_{0}<1$ and then for each $t<t_{0}$ choose one in the following manner: if $u_{1}, \cdots, u_{k(t)}$ are the base points chosen for the cover of 
$E_{t}$, and if $t^{\prime}<t^{\prime \prime}<t_{0}$, then $\left\{u_{1}, \cdots, u_{k\left(t^{\prime}\right)}\right\} \supset\left\{u_{1}, \cdots, u_{k\left(t^{\prime \prime}\right)}\right\}$. Let $\omega_{t}=\bigcup_{j=1}^{k(t)} \Gamma_{\alpha}\left(u_{j}\right)^{2}$.

LEMMA 2. $\int_{\omega \alpha(E)}|\nabla f|^{2} d \mu(z)<\infty$.

We prove this by first applying Green's theorem to $\omega_{t}$. Then, using the estimates of Lemma 1 translated by the group $N$ and the uniform boundedness of $f$, we obtain $\int \omega_{t}|\nabla f|^{2} d \mu(z) \leqq k \int_{\partial \omega_{t}} d s$ when $k$ is independent of $t$. Now we let $t$ tend to 0 , and observe that $\int_{\partial \omega_{t}} d s \leqq M$ independently of $t$. Part (a) then follows from:

LEMMA 3. Suppose $E \subset B$ is compact and $f$ is locally bounded and positive in D. If $\int_{\omega_{\alpha}(E)} f d \mu<\infty$, then $\int_{\Gamma_{\beta}(u)} h(z)^{-n} f(z) d \mu(z)<\infty$ for all $\beta>0$ and almost every $u \in E$.

We now outline the proof of part (b).

LEMMA 4. If $\int_{\Gamma_{\alpha}(0)} h(z)^{-n}|\nabla f|^{2} d \mu(z)<\infty$, then $h(z)\left|\partial f / \partial z_{1}\right|$ and $h(z)^{1 / 2}\left|\partial f / \partial z_{k}\right|, k \geqq 2$, are bounded in $\Gamma_{\alpha^{\prime}}(0)$ for $\alpha^{\prime}<\alpha$.

To prove this let

$$
\begin{aligned}
D_{1} & =\frac{\partial}{\partial z_{1}}+\frac{\partial}{\partial \bar{z}_{1}} \\
D_{k} & =2 i z_{k} \frac{\partial}{\partial z_{1}}+\frac{\partial}{\partial \bar{z}_{k}}, \\
D_{k^{\prime}} & =-2 i \bar{z}_{k} \frac{\partial}{\partial \bar{z}_{1}}+\frac{\partial}{\partial z_{k}}, \\
D_{0} & =z_{1} \frac{\partial}{\partial z_{1}}+\bar{z}_{1} \frac{\partial}{\partial \bar{z}_{1}}+\frac{1}{2} \sum_{2}^{n}\left(z_{k} \frac{\partial}{\partial z_{k}}+\bar{z}_{k} \frac{\partial}{\partial \bar{z}_{k}}\right) .
\end{aligned}
$$

We then observe that if $f$ is harmonic then $D_{0} f, D_{1} f, D_{k} f, D_{k}, f$ are harmonic, and thus can be represented as Poisson integrals. Now $|\nabla f|^{2}$ dominates $h\left|D_{1} f\right|^{2},\left|D_{k} f\right|^{2},\left|D_{k^{\prime}} f\right|^{2}$ and $h^{-1}\left|D_{0}\right|^{2}$ in $\Gamma_{\alpha}(0)$; and the latter dominate $h\left|\partial f / \partial z_{1}\right|^{2}$ and $\left|\partial f / \partial z_{k}\right|^{2}$ for $k \geqq 2$, in $\Gamma_{\alpha}(0)$. Now, using Green's theorem and Lemma 4, we have

$$
\int_{\partial_{\omega t}} f^{2} d s \leqq k \int_{\partial_{\omega t}}|f| d s+k^{\prime} \int_{\omega t}|\nabla f|^{2} d \mu .
$$

LEMma 5. Suppose $E \subset B$ is compact, $f$ is nonnegative and locally bounded in $D$, and for each $u \in E$, there exists an $\alpha>0$ such that $\int_{\Gamma_{\alpha}(u)} f d \mu<\infty$. Then for every $\epsilon>0$ and $\beta>0$ there exists a compact set $F \subset E$ such that meas $(E \backslash F)<\epsilon$, and $\int_{\partial \omega_{\beta}(F)} h(z)^{n} f(z) d \mu(z)<\infty$. 
Applying this to the inequality above we have $\int_{\partial \omega_{t}} f^{2} d s \leqq M$ independently of $t$. Now a standard argument (see Stein [5]) shows that $|f(z)| \leqq c g(z)+c^{\prime}$ in $\omega_{\alpha}(E)$ where $g$ is the Poisson integral of some function in $L^{2}(B)$. The result now follows from Koranyi [2].

\section{REFERENCES}

1. A. P. Calderon, On a theorem of Marcinkiewicz and Zygmund, Trans. Amer. Math. Soc. 68 (1950), 55-61. M R 11, 357.

2. A. Korányi, Harmonic functions on hermitian hyperbolic space, Trans. Amer. Math. Soc. 135 (1969), 507-516.

3. J. Marcinkiewicz and A. Zygmund, A theorem of Lusin, Duke Math. J. 4 (1938), 473-485.

4. D. C. Spencer, A function-theoretic identity, Amer. J. Math. 65 (1943), 147-160. MR 4, 137.

5. E. Stein, On the theory of harmonic functions of several variables. II: Behavior near the boundary, Acta Math. 106 (1961), 137-174. MR 30 \#3234.

WASHINGTON UNIVERSITY, St. LoUIS, MisSOURI 63130 